\title{
Development of methodical instruments of obtaining, research and control of electrophysical service properties of nanostructure composites
}

\author{
Gennady K. Baryshev ${ }^{1, *}$, Anastasia S. Kondratyeva ${ }^{2}$, and Aleksandr V. Berestov ${ }^{1}$ \\ ${ }^{1}$ National Research Nuclear University MEPhI (Moscow Engineering Physics Institute), Russian Federation \\ ${ }^{2}$ National Research University St. Petersburg State Polytechnical University, Russian Federation
}

\begin{abstract}
The article highlights the main problems of development of methodical instruments of obtaining, research and control of electrophysical service properties of nanostructure composites. Some of the results obtained at the research performed by scientists from National Research Nuclear University MEPhI (Moscow Engineering Physics Institute) and National Research University St. Petersburg State Polytechnical University are presented. The results are discussed in the focus of development of a new general method of control of electrophysical service properties of materials and products of nanostructured composites during the manufacturing process.
\end{abstract}

\section{Introduction}

The national problem of rapid development of Russian technologies and manufacturing of new competitive products for the purpose of import substitution is actual at the moment $[1,2]$. These new products could be manufactured of nanostructured composites in various areas - nuclear and space industries, perspective markets of the National Technology Initiative, etc.

Nickel oxide, for example, is related to the class nanostructured composites, applied as a high-sensible and selective oxide sensor. Such sensors could find their application in systems of monitoring of the environment, medical diagnostic devices, etc.

Semiconductor Heusler alloys appeared very promising thermoelectric materials as well, with better service properties - physical quantities thermal emf coefficient, conductivity, and thermal conductivity, than traditional thermoelectric materials [3].

Another class of nanostructured materials that is of significant interest are electro technical materials, such as composite wires for power lines, operating in severe climatic conditions and other applications [4].

The set of methods of manufacturing of materials and products with the given operating conditions, including products of nanostructured composites, is rapidly growing at the moment. At the same time, most of these methods are at the research and testing stage, and samples and prototypes of nanostructured composites need general industrial methods and methodical instruments of obtaining, research and control of their service properties, including electrophysical service properties, to be developed. In this paper we describe some of the results obtained by our research group in this area on such materials of nanostructured composites as layers of ferrous-group metals and composite powders.

\section{Development of methodical instruments of obtaining layers of ferrous-group metals}

First, we will provide some information on the perspective technology and instruments of obtaining layers of ferrous-group metals related to the class of nanostructured composited with wide perspectives of application.

Thin layers of ferrous-group metals have a set of properties such as electrochromism, sensor activity, magnetic properties. One of the most attractive methods of obtaining these layers is the process of chemical sedimentation from gas phase that provides formation of high-quality layers on samples of complex form. To provide this the technology of chemical sedimentation of thin layers of ferrous-group metals from gas phase is developed, physical and chemical principles of the process are investigated, as well as practical properties of layers.

It is essential that for the last decades most innovations realized in high-technology economics sector and industry are based on application of engineering technologies and new materials. Thereafter, the so called "smart materials" - a unique class of materials which properties change under external factor in a specific way we expect them to change - are in most of interest thereafter.

Russian Federation is now in the active phase of the process of industrial modernization and development of digital economics. Major part of industrial equipment

\footnotetext{
*Corresponding author: GKBaryshev@mephi.ru
} 
has been in operation since the Soviet Union times. Still, what the industry need first of all are analytical devices and systems. Thus we may state that a market of chemical semiconductor sensors is a perspective economic niche in Russia.

The most known worldwide oxide semiconductor sensor producers are City Technology, Alphasense, Applied Sensor and Figaro Inc. Russian companies hold a very little percent of the market, being mostly at the stage of research and development. The years of 20202025 may be the time of total implementation of MEMC-technology sensors, with Russian companies being outside this technological trend, if they stay where they are, with the current level of research and development [5]. The growth of non-military purpose sensors market would be increasing rapidly in the nearest decade.

Most perspective materials to be applied in gas sensors are ferrous-group metals oxides. The surface of material is modified in the interaction with the environment. The application of semiconductor oxide layers as a high-sensitive and selective sensors is based on this effect [6,7]. Sensors of this kind can detect hydrogen, nitrogen oxide, carbon monoxide, ammonia, organic materials vapors such as acetone, formaline, methanol. They are used in systems of continuous environmental monitoring, fire alarms, medical diagnostics devices, catalysts for organic synthesis of biomass pyrolysis in bioenergetics [8].

Besides, thin layers of ferrous-group metals have a feature of electrochromism [7] - an ability to change color at the process of electron extraction, that can be used, for example, in energy-saving monitors. On the base of these materials chemically compatible capacitor electrodes for oxide isolators and conductive transparent electrodes for optical devices (light- and photo-diodes, computer monitors etc.) can be produced $[6,7,9]$.

Two most commonly used methods to obtain layers of ferrous-group metals are magnetron sputtering in oxide atmosphere and sol-gel synthesis. The main limitation of the first one is inability to control the morphology of obtained layers, for the second one this is a large amount of admixtures in the product and inability to control the material structure in large scales. This way we consider a perspective method for obtaining thin layers of different materials the chemical sedimentation from gas phase that has a number of advantages [9]: high growth velocities, simplicity and low price of equipment, availability to control the product features by the parameters of the process (temperature, partial pressure of reagents, resident time). One of the key advantages of the chemical sedimentation from gas phase technology is easiness of technology scaling to mass-production.

We will now describe the technology more thoroughly. To solve a specific task we need a layer of material with given properties. Research of physical and chemical laws of chemical sedimentation from gas phase would allow us to develop certain engineering and industrial technologies [10].
First, one needs to study the process of gas-phase transforming in the Cp2Me-O2-Ar and Cp2Me-O2-O3Ar systems, this would allow to:

1.Formulate a model of chemical reactions and processes of obtaining the layers of ferrous-group metals in the systems;

2. Estimate how sedimentation temperature influences the process;

3. Estimate the oxidant influence in the system.

It is also important to study the influence of main technological parameters of the $\mathrm{Cp} 2 \mathrm{Me}-\mathrm{O} 2-\mathrm{Ar}$ and $\mathrm{Cp} 2 \mathrm{Me}-\mathrm{O} 2-\mathrm{O} 3-\mathrm{Ar}$ systems on sedimentation velocity, this would allow one to estimate the time of the process limiting stage. This part includes studying of how the following technological parameters influence the layer sedimentation velocity:

1. Active zone temperature;

2. Active zone pressure;

3. General velocity of gas flow;

4. Supporting plate type (including crystallographic orientation);

5. Partial pressure of reagents.

It is a feature of such systems for chemical sedimentation to pass both in kinetic and diffusion modes, depending on the specific parameter values, with the kinetic mode transforming into the diffusion one when temperature increases.

The complex study of the layers obtained (chemical composition, morphology, crystallographic structure) would allow to get most complete data about their properties. Such data obtained would be the physical basis for the development of industrial technology.

\section{Developing methodical instruments of research and control of electrophysical properties of composite powders at cold pressing}

Another significant problem is developing the necessary methodical instruments of control of electrophysical properties of composite powders at cold pressing.

According with the practice of obtaining coldpressed powders in various matrixes the heterogeneity of the density at the height of the backfill is considerable if ratio of the diameter to height of the matrix is less than $1: 10$. This manufacturing research problem could be solved by the development of specific methodical instruments: information measuring system for monitoring and control of the changes of porosity during the pressing process. Special attention to the development of mobile sensors should be paid when designing and developing controls of informationmeasuring and information-analytical systems for that purpose.

Automation and effective management of powder processing requires designing of positioning system of sensors, that monitoring filling level, and obtaining data of formed pressed powder. Data collection should be 




Fig. 1. The hierarchy of the virtual instrument

carried out by programmable logic elements included into information-analytical system [11].

Software development for solving task was implemented by NI LabVIEW 8.6.

A sensor-positioning system based on method of electrical resistance measuring was developed. The main sensors installed on the mobile carriage are developed to provide automation of the measurement and control of porosity during the process of cold pressing. Depending on the height of the used backfill measuring circuit may include one or more sensors.

Kinematic scheme of positioning mechanisms, as a rule, has two main parts: the operating mechanism (or sensor) and its actuator. The actuator's carriage serves as base frame for sensor by moving along linear guideway. To achieve the necessary dynamic quality of the positioning mechanism and also to meet the requirements of interchangeability the carriage should satisfy the following requirements [12]:

- ease of movement with no backlash and clearances;

- high precision movement;

- low weight;

- high rigidity;

- convenience for installation and alignment

These requirements can be met by choosing the appropriate engineering materials of the carriage, its geometric shapes, precision linear guides with ball bearings, design of their fabrication, providing a preload to the base side of the guide. Switching on the actuator will be blocked in case of lack of tightness of the measuring mandrel or opened top cover.

Because the height of the filling powder decreases at the process of pressing, it is necessary to use a system, which includes the height level backfill sensor (or density sensor), to work jointly with the positioning system of the carriage.
The dimensional difference for the carriage positioning must not exceed $10-20 \mathrm{mcm}$. The chosen positioning scheme is similar to that of applied for computer floppy discs. We use the stepping engine as a driver to move the carriage with the aid of an actuator screw. Such a system does not have high dimanic parameters but is still characterized by relative simplicity and high level of reliability. The controller of powder level height is integrated into the carriage control system. To control positioning the computer program has setting commands. For example, the "set the sensor" command is applied to move the carriage with the sensor to the given position and connect it with the measuring device. Search commands are applied for control sensors as well as for database operating. The latest procedure is necessary for selecting a sample group of data relative to the same powder height level, comparing averaged readings for different powder level height or comparing data for different powders etc. The recording commands group includes commands performing both data recording as well as recording the control information (personal address, numerical regions, keys). For example, for recording the data obtained into a file the "record data" command is applied. This command is to go after the search commands (searching for the identifier or key equality). One may perform the command in case the data array to be written fits the assigned memory volume. The operating commands include such commands as "check input-output", "confirm condition" and a number of others.

By method of electrical resistivity we can estimate the porosity of powder depending on the applied pressure. Measurement of the powder electrical resistance was carried out using a high-performance digital multimeter Agilent 34401A according to two methods: wire and potentiometric circuits. According to user manual multimeter Agilent 34401A could provide measurement of the resistance by using two-wire and 
four-wire circuits. In both cases electric current flows from high-potential nest HI through the test sample.

We also have developed the information measuring system for registration diagnostic signals, which includes the protocol of data exchange between PC, digital multimeter and virtual device (Fig 1.)., that displays on the monitor screen current and the integral values of the resistance (Fig. 2).

Further improvement of measurement tools and methods by using the achievements of modern information technologies will allow achieving a greater sensitivity for measuring thickness of research and control of electrophysical properties of composite powders at cold pressing.



Fig. 2. The outer panel of the virtual instrument

\section{References}

1. Strategy of the National Security of Russian Federation till 2020, approved by the Decree of the President of Russian Federation of May $12^{\text {th }} 2009$ \#537

2. Decree of the Government of Russian Federation of April 15 2014 \#328 "About the Approving of the State Program of Russian Federation "Development of the Industry and Increasing of Its Competitiveness"

3. A.I. Voronin, G.K. Baryshev, Yu. V. Bozhko, A.A. Usenko, V. Yu. Zueva, K.I. Litvinova, I.V. Petrova, M.A. Seredina, V.V. Hovailo, Bulletin of the Lebedev Physics Institute 42, 7 (2015)

4. A. V. Putilov, A. K. Shikov, V. I. Pantsyrny, A. E. Vorobieva, V. A. Drobyshev, Tsvetnye Metally (Non-ferrous Metals) 3 (2008)

5. V.V. Zheltova, Saint-Petersburg National Research University of Information Technologies, Mechanics and Optics (2013)

6. Jin-Kyu Kang, Shi-Woo Rhee, Thin Solid Films 391 (2001)

7. B. Subramanian, M. Mohammed Ibrahim. J. Mat. Sci: Mater. Electron 20 (2009)

8. Jianfen Li, Rong Yan, Bo Xiao, David Tee Liang, Dong Ho Lee, J. Am. Chem. Soc. (2008)
9. Pavel Moravec, Jiří Smolík, Helmi Keskinen, Jyrki M. Mäkelä, Snejana Bakardjieva, Valeri V. Levdansky, Mat. Sci. and App. 2 (2011)

10. Gennady K. Baryshev, Anastasia S. Kondratyeva, Proceedings of 2016 International Conference on Control, Instrumentation, Communication and Computational Technologies (ICCICCT) (2016)

11. Aleksandr P. Biryukov, Gennady K. Baryshev, Vitaliy I. Surin, Yuri Yu. Vostrenkov, Proceedings of 2016 13th International Scientific-Technical Conference on Actual Problems of Electronic Instrument Engineering (APEIE) (2016)

12. G.K. Baryshev, E.A. Olevsky, V.I. Surin, A.I. Maksimkin, Information technologies in design and manufacturing 2 (2013) 